\title{
Renegotiation in Repeated Games with Side-Payments ${ }^{1}$
}

\author{
Sandeep Baliga \\ Kellogg Graduate School of Management, MEDS, 2001 Sheridan Road, \\ Evanston, Illinois 60208-2009 \\ E-mail: baliga@nwu.edu \\ and

\section{Robert Evans} \\ St. John's College, Cambridge, CB2 1TP, United Kingdom \\ E-mail: robert.evans@econ.cam.ac.uk
}

Received March 27, 1998

\begin{abstract}
We consider repeated games with side-payments: players have an endowment of wealth in each period in which transfers can be made. We show that if endowments are large enough and the common discount factor high enough, then a strongly renegotiation-proof equilibrium (SRP) in the sense of Farrell and Maskin exists. As the discount factor goes to 1, the set of SRP payoffs converges to the set of efficient, individually rational payoffs. These results provide a justification for the efficiency principle when agreements are not enforceable. Journal of Economic Literature Classification Numbers: C73, D23, L14. ๑ 2000 Academic Press
\end{abstract}

\section{INTRODUCTION}

A large part of the modern economic analysis of organizations is based on the efficiency principle, which is summarized in the following terms by Milgrom and Roberts (1992, p. 24): "If people are able to bargain together effectively and can effectively implement and enforce their decisions, then the outcomes of economic activity will tend to be efficient (at least for the parties to the bargain)." For applications of this principle, in organization theory and in other branches of economics, see, for example, Hart (1995),

\footnotetext{
${ }^{1}$ We are grateful for helpful comments by Eric Maskin, Stephen Morris and an anonymous referee. 
Greif (1989), Milgrom et al. (1990), and Rotemberg and Saloner (1986). More particularly, if there are no wealth effects (that is, if utility functions are quasi-linear) then one can appeal to the value maximization principle (Milgrom and Roberts, 1992, p. 36), which asserts that an allocation is efficient only if it maximizes the total value to the affected parties, and the Coase theorem, which says that value-creating activities which result from bargaining will be determined entirely by efficiency and not by relative bargaining powers.

One crucial assumption on which these principles depend is that decisions are enforceable. In many situations of interest the only effective means of enforcement derives from the repetition of the relationship in question. In the context of a repeated relationship the logic of the efficiency principle, which is that people will negotiate an efficient agreement if their ex ante position is inefficient, suggests that they will renegotiate this agreement if it ever leads them to an inefficient position. Hence the notion of renegotiation-proof equilibrium of a repeated game. The question then naturally arises: if, in a repeated game, utilities are quasi-linear in money or some other transferable good, do renegotiation-proof equilibria satisfy the efficiency principle and the Coase theorem?

Several solution concepts for repeated games have been proposed to incorporate renegotiation (Abreu et al. (1993), Asheim (1991), Bernheim and Ray (1989), Pearce (1987)). In this paper, we will focus only on those of Farrell and Maskin (1989):

(1) Weakly renegotiation-proof equilibrium: a subgame perfect equilibrium is weakly renegotiation-proof (WRP) if, given any two continuation equilibria, neither Pareto-dominates the other; and

(2) Strongly renegotiation-proof equilibrium: a subgame perfect equilibrium is strongly renegotiation-proof (SRP) if it is WRP and none of its continuation equilibria is dominated by another WRP equilibrium.

The idea behind WRP equilibrium is as follows. Suppose that the two players make an agreement, or contract, to play the game in a certain way (perhaps in a Pareto-efficient way), giving long-run payoff pair $\left(v_{1}, v_{2}\right)$. We assume that this agreement must be self-enforcing, implying that it must at least be subgame-perfect. However, it should also be self-enforcing in a collective sense. The contract will need to specify what happens if one party violates the agreement and it may be, for example, that in that event the continuation payoff pair $\left(\tilde{v}_{1}, \tilde{v}_{2}\right)$ is Pareto-dominated by $\left(v_{1}, v_{2}\right)$. The two parties would then want to let bygones be bygones and renegotiate to the Pareto-superior continuation $\left(v_{1}, v_{2}\right)$, which, by assumption, is a credible agreement for them to make. This argument would then establish that the original agreement is in fact not credible. The idea of SRP equilibrium is that if every WRP equilibrium is regarded as a credible contract then one 
should allow the parties, after any history, to renegotiate by substituting a Pareto-superior WRP agreement, if there is one, for the current contract. Again, if this is ever possible, the original agreement is not credible.

It is straightforward to show that many WRP equilibria do not satisfy the efficiency principle; indeed, in many games, such as the prisoners' dilemma and Cournot duopoly, any individually rational payoff vector can arise in WRP equilibrium. The concept is clearly, therefore, too weak for our purposes. What of the stronger concept, SRP equilibrium? In general games (in which side-payments are not necessarily available) SRP equilibria may well be inefficient. Moreover, it turns out that SRP equilibria do not always exist. For example, if the discount factor is high enough, SRP equilibria exist in the prisoners' dilemma and Cournot duopoly but not in Bertrand duopoly. Roughly speaking, an SRP equilibrium can fail to exist because it may happen that, in the game in question, any WRP equilibrium on the Paretoboundary of the set of WRP equilibria has a 'normal' phase which must be supported by 'punishment' phases with continuation equilibrium payoffs which are Pareto-dominated by those for some other WRP equilibrium.

If players have transferable wealth they have the possibility of stipulating fines for non-compliance. We show in this paper that if that is the case, and the players have quasi-linear utility, (i.e., in the circumstances postulated by the Coase Theorem) then (a) SRP equilibrium exists if the wealth endowments are large enough and the players are patient enough, and (b) if the players are patient, all SRP equilibrium payoffs are approximately efficient. Thus, versions of the efficiency principle and the Coase Theorem hold when agreements have to be self-enforcing. The notion of renegotiation-proofness cannot, however, be used to select from among efficient payoffs: any strictly individually rational, efficient payoff is SRP if the discount factor is high enough.

We confine attention to two-player games: in larger games, renegotiation by proper subsets of players would have to be considered. In Section 2, we introduce our model and Section 3 contains our results.

\section{THE FRAMEWORK}

\subsection{The One-Shot Game with Transfers}

Consider a two-person finite game $g: S_{1} \times S_{2} \rightarrow \Re^{2}$, where $S_{i}(i=1,2)$ is player $i$ 's pure action set and $g_{i}\left(s_{1}, s_{2}\right)$ is $i$ 's payoff. We let $A_{i}$ denote player $i$ 's set of mixed actions. The set of payoffs, allowing for convexification ${ }^{2}$ as

${ }^{2}$ Any payoff pair in the convex hull of the set of pure strategy payoff pairs can be achieved in the infinitely repeated game, if the discount factor is high enough, without public randomization. See Farrell and Maskin (1989). 
well as mixed strategies, is

$$
V:=\operatorname{co}\left(\left\{\left(v_{1}, v_{2}\right) \mid \exists\left(a_{1}, a_{2}\right) \in A_{1} \times A_{2} \text { such that } g\left(a_{1}, a_{2}\right)=\left(v_{1}, v_{2}\right)\right\}\right),
$$

where "co" denotes the convex hull and $g\left(a_{1}, a_{2}\right) \equiv\left(g_{1}\left(a_{1}, a_{2}\right), g_{2}\left(a_{1}, a_{2}\right)\right)$ denotes the expected payoff pair resulting from the play of the mixed strategies $\left(a_{1}, a_{2}\right)$. We normalize min-max payoffs, $\min _{a_{j} \in A_{j}} \max _{a_{i} \in A_{i}} g_{i}\left(a_{i}, a_{j}\right)$ $(j \neq i)$, to zero. Let $i$ 's "cheating payoff," given mixed action pair $a \equiv$ $\left(a_{i}, a_{j}\right)$, be $c_{i}(a):=\max _{s_{i} \in S_{i}} g_{i}\left(s_{i}, a_{j}\right)(i=1,2) . c_{i}(a)$ is the highest payoff that player $i$ can get in the one-shot game if the 'agreed' action pair is $a$ and $i$ breaks this agreement.

Assumption 1. There exists a unique action-pair $s^{*} \equiv\left(s_{1}^{*}, s_{2}^{*}\right) \in S_{1} \times S_{2}$ that maximizes the sum of payoffs in $V$. That is, $v^{*} \equiv g_{1}\left(s^{*}\right)+g_{2}\left(s^{*}\right) \geq$ $g_{1}(s)+g_{2}(s) \forall s \in S_{1} \times S_{2}$, with the inequality holding strictly if $s \neq s^{*}$.

This assumption holds generically in games with a finite number of pure actions. Let $v_{i}^{*}:=g_{i}\left(s^{*}\right)(i=1,2)$. Thus, $v^{*}=v_{1}^{*}+v_{2}^{*}$. We make the further assumption that, when the efficient action $s^{*}$ is played, both players get strictly more than their min-max payoff (this is not necessary for our results, but it slightly simplifies the argument):

Assumption 2. $v_{i}^{*}>0(i=1,2)$.

We depart from the standard framework in the following way. We assume that in each period each player gets an endowment ${ }^{3} e$ of a perishable $e^{4}$ good and he or she can transfer all or part of it to the other player. Player $i$ 's utility function is $u_{i}\left(s_{1}, s_{2}, y_{i}\right):=g_{i}\left(s_{1}, s_{2}\right)+\theta_{i} y_{i}$, where $y_{i}$ is $i$ 's consumption of the transferable good and $\theta_{i}$ is a strictly positive constant. Without loss of generality, we normalize ${ }^{5}$ utilities so that $\theta_{1}=\theta_{2}=1$. We denote player $i$ 's transfer of the good to player $j$ by $f_{i}$, where $0 \leq f_{i} \leq e$. If the players play the pure strategy pair $\left(s_{1}, s_{2}\right), 1$ transfers $f_{1}$ to 2 and 2 transfers $f_{2}$ to 1 then player $i$ 's net payoff, $g_{i}^{\prime}\left(s_{1}, s_{2}, f_{1}, f_{2}\right)$, is given by $g_{i}\left(s_{1}, s_{2}\right)+e+f_{j}-f_{i}$. We shall need to assume that the per-period wealth level is large relative to the payoffs available in the game $g$. Specifically,

$$
\text { Assumption 3. } e>3 \max _{s \in S_{1} \times S_{2}, i=1,2}\left|g_{i}(s)\right| \text {. }
$$

${ }^{3} \mathrm{We}$ assume that both players have the same endowment only for simplicity.

${ }^{4}$ The assumption that the good is perishable is needed to ensure that there is no borrowing and saving between periods. Otherwise, the game would not be stationary and we could not use the standard techniques of the theory of repeated games or the standard definitions of WRP and SRP equilibrium.

${ }^{5}$ The normalization of $g$ which sets min-max payoffs to zero is achieved by adding a (possibly different) constant to each player's payoff; this second normalization involves scaling, and preserves each player's min-max payoff in $g$ as zero. 


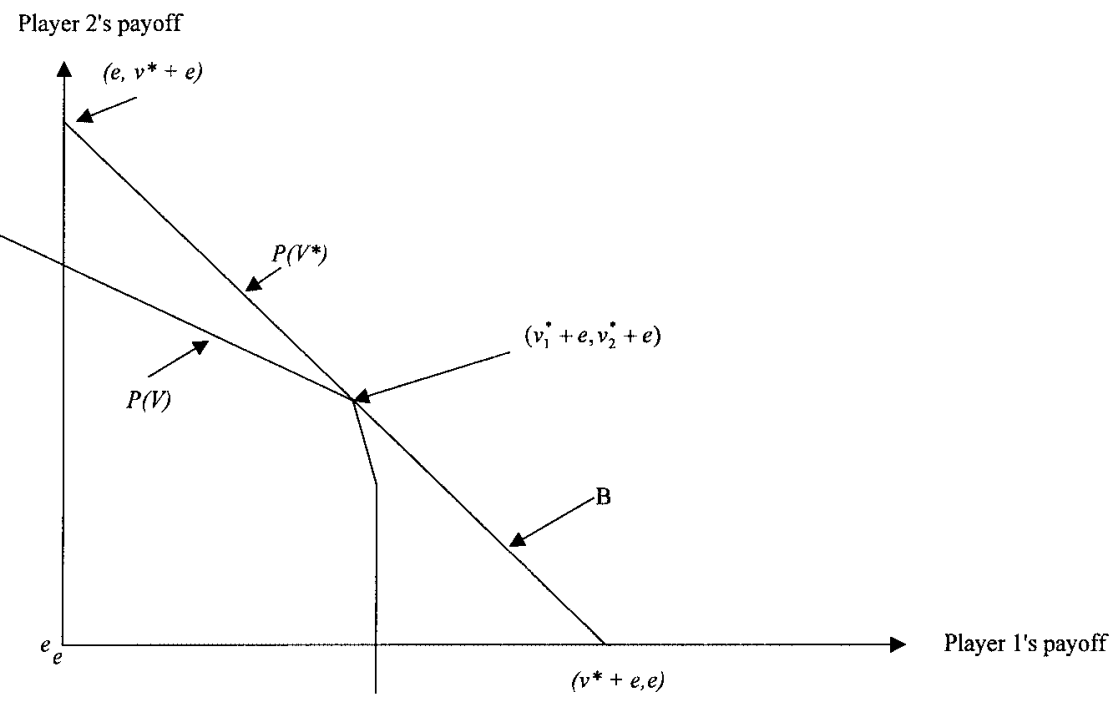

FIGURE 1

Let $S_{i}^{\prime}:=S_{i} \times[0, e]$ and let $A_{i}^{\prime}$ be the set of probability measures on $S_{i}^{\prime}$. A pure action in the modified game $g^{\prime}$ is a pair $\left(s_{i}, f_{i}\right)$ in $S_{i}^{\prime}$ and so a mixed action $a_{i}^{\prime}$ is an element of $A_{i}^{\prime} \cdot g_{i}^{\prime}\left(a^{\prime}\right)$ is defined in the obvious way. In $g^{\prime}$, each player has a min-max payoff of $e$. This is because if $i$ wants to min-max $j, i$ should play an action, $a_{i}^{m}$, which is a min-max action of $g$ and make no transfer to $j$, while $j$ should play a best reply to $a_{i}^{m}$ and make no transfer to $i$. Let $c_{i}^{\prime}\left(a_{1}^{\prime}, a_{2}^{\prime}\right)$ be $i$ 's cheating payoff given action pair $\left(a_{1}^{\prime}, a_{2}^{\prime}\right)$. The mixed action $a_{j}^{\prime}$ has a marginal distribution, $a_{j}$, over $S_{i}$. Because of the quasi-linear form of the payoffs, $i$ should, if he wants to achieve $c_{i}^{\prime}\left(a_{1}^{\prime}, a_{2}^{\prime}\right)$, play a best response in $g$ to $a_{j}$ and make no transfer to $j$.

Finally, let

$$
V^{\prime}:=\operatorname{co}\left(\left\{\left(v_{1}, v_{2}\right) \mid \exists\left(a_{1}^{\prime}, a_{2}^{\prime}\right) \in A_{1}^{\prime} \times A_{2}^{\prime} \text { such that } g\left(a_{1}^{\prime}, a_{2}^{\prime}\right)=\left(v_{1}, v_{2}\right)\right\}\right)
$$

and let $V^{*}:=\left\{\left(v_{1}, v_{2}\right) \in V^{\prime} \mid v_{1}>e, v_{2}>e\right\}$. $V^{\prime}$ represents feasible payoff pairs and $V^{*}$ individually rational feasible payoff pairs, when transfers are allowed. The closure of the Pareto frontier of $V^{*}$ is denoted by $P\left(V^{*}\right)$; the Pareto frontier of $V$ is denoted by $P(V)$ (see Fig. 1$)^{6}$.

\subsection{The Repeated Game}

The repeated game $g^{*}$ is the game $g^{\prime}$ played in each of infinitely many periods $t=1,2, \ldots$. In period $t$, let $a_{i}^{\prime}(t)$ be the mixed action taken by

${ }^{6}$ The point on the Pareto frontier denoted $B$ will be utilized in the exposition below. 
player $i(i=1,2)$. A strategy $\sigma_{i}(i=1,2)$ for player $i$ is a function that for every $t$ and every possible history $\left(a_{1}^{\prime}(1), a_{2}^{\prime}(1) ; \ldots ; a_{1}^{\prime}(t-1), a_{2}^{\prime}(t-1)\right)$ defines a period $t$ action $a_{i}^{\prime} \in A_{i}^{\prime}$. This formulation embodies the assumption, made for simplicity, that mixed strategies are observable. Given $\left\{\left(a_{1}^{\prime}(\tau), a_{2}^{\prime}(\tau)\right)\right\}_{\tau=1}^{\infty}$, a sequence of mixed actions in $g^{\prime}$, we define the average expected payoff to player $i$ as $(1-\delta) \sum_{\tau=1}^{\infty} g_{i}^{\prime}\left(a_{1}^{\prime}(\tau), a_{2}^{\prime}(\tau)\right) \delta^{\tau-1}$ where $\delta \in(0,1)$ is the discount factor, common to both players.

A pair of strategies $\sigma \equiv\left(\sigma_{1}, \sigma_{2}\right)$ defines a probability distribution on infinite histories and therefore on payoffs; we write $g^{*}(\sigma, \delta)$ for the expected average payoffs when the discount factor is $\delta$ and players follow the strategies $\sigma$. It is the payoff function for the game $g^{*}$. The strategy profile $\sigma \equiv\left(\sigma_{1}, \sigma_{2}\right)$ is a Nash equilibrium of $g^{*}$ if $\sigma_{1}$ is a best response to $\sigma_{2}$ and vice versa; it is also a subgame perfect equilibrium if the subgame strategies induced by $\sigma$ in every subgame are Nash equilibrium strategies.

\section{DEFINITIONS AND RESULTS}

We begin by defining the notions of renegotiation-proof equilibrium that we employ. They are taken from Farrell and Maskin (1989).

DEFINITION 1. A subgame-perfect equilibrium $\sigma$ is weakly renegotiation-proof (WRP) if there do not exist continuation equilibria $\sigma^{1}, \sigma^{2}$ of $\sigma$ such that $\sigma^{1}$ strictly Pareto-dominates $\sigma^{2}$. If an equilibrium $\sigma$ is WRP then we also say that the average payoffs $g^{*}(\sigma, \delta)$ are WRP.

The idea behind this, as noted above, is that if $\sigma^{1}$ and $\sigma^{2}$ are both continuation equilibria then they can both be regarded as possible ways of playing the game. This implies that if $\sigma^{1}$ dominates $\sigma^{2}$ then the players should, when the equilibrium specifies that $\sigma^{2}$ be played, renegotiate and play $\sigma^{1}$ instead. This would invalidate $\sigma$ as a renegotiation-proof equilibrium.

DEFINITION 2. $\sum_{\delta}$ is the set of all WRP equilibria for discount factor $\delta$ and $W(\delta):=g^{*}\left(\sum_{\delta}, \delta\right) . P(W(\delta))$ is the weak Pareto frontier of $W(\delta)$.

In general there will be many WRP equilibria. Furthermore, while no continuation of a WRP equilibrium can be Pareto-dominated by another continuation of the same equilibrium, it may well be Pareto-dominated by a continuation of another WRP equilibrium. If every WRP equilibrium is regarded as a possible way of playing the game, this opens up the possibility of renegotiation from one WRP equilibrium to another. That is, WRP equilibrium may be too weak a concept. This leads to the next concept. 
DEFINITION 3. A WRP equilibrium is strongly renegotiation-proof (SRP) if none of its continuation equilibria is strictly Pareto-dominated by another WRP equilibrium.

The point of view taken in this paper is that the two players are able to communicate with one another and will negotiate an efficient agreement $\sigma$ if a credible one can be found. By 'credible' we mean (a) that neither party will, given the sanctions specified in the agreement $\sigma$, want to deviate unilaterally from it after any history (i.e., $\sigma$ is subgame-perfect), (b) that the parties cannot, after any history, both improve their expected payoff by substituting a different continuation of $\sigma$ (i.e., $\sigma$ is WRP) and (c) that if, after some history, there is some other feasible agreement $\sigma^{\prime}$ which is subgame-perfect and which strictly Pareto-dominates $\sigma$ from this point on, then $\sigma^{\prime}$ is not WRP, so that the renegotiation from $\sigma$ to $\sigma^{\prime}$ would itself not be credible. In other words, $\sigma$ must be SRP. The question we ask is does there exist an efficient SRP equilibrium?

One might object to the SRP equilibrium concept on a number of different grounds. Firstly, it can be argued that it is too strong a concept: for example, one might argue that an equilibrium $\sigma$ which, after some history, is Pareto-dominated by a WRP equilibrium $\sigma^{\prime}$, will not be renegotiated to $\sigma^{\prime}$ if $\sigma^{\prime}$ is itself not credible for some other reason (perhaps $\sigma^{\prime}$ is, after some subsequent history, Pareto-dominated by another WRP equilibrium $\tilde{\sigma})$. On the other hand, our results below establish that, in our context, SRP equilibrium always exists (for high $\delta$ ) and that any efficient payoff pair can be achieved in some SRP equilibrium (again, for high $\delta$ ). Therefore the strength of the concept is an advantage. Secondly, in the efficient SRP equilibria which we construct it typically happens that if a player deviates (breaks the agreement) then that player is punished for some period in two ways: the two players play actions which are unfavorable to the deviator and the deviator has to pay a fine to the other player. At the start of this punishment phase, it may be that the deviator's continuation payoff is very low and the other player's is very high. It could be argued that at this juncture the payoffs are so asymmetric that the punished player might not want to continue with the agreed strategy (he might, say, refuse to pay the fine). Since the equilibrium is subgame-perfect the deviator's continuation payoff, low as it is, is still higher than the payoff which he would get if he were to refuse to pay the fine; it might be, however, that the punished player might give up some payoff in order to counteract the perceived unfairness of the equilibrium. On the other hand, it is not clear that the asymmetry is unfair if it has arisen only because the player has violated an agreement which he voluntarily entered into. In any event, we take the conventional game-theoretic view that players simply act so as to maximize their expected payoffs. Another objection which might be made is 
that, given the asymmetry of the payoffs in a punishment phase, the players might prefer an equilibrium which is Pareto-inefficient but which requires no punishment (say, a Nash equilibrium of the stage game which has payoffs which are almost efficient). In a context in which the players face a small amount of noise this would be true. Here, though, we are considering a complete information game. Since each player is only interested in maximizing his or her own expected payoffs, and since the event of a punishment phase has zero (or vanishingly small) probability, they will both prefer a Pareto-superior efficient agreement, notwithstanding the risk of incurring a punishment.

The set $V^{*}$ of feasible, individually rational payoffs is bounded by the axes $\left\{v_{1}=e\right\}$ and $\left\{v_{2}=e\right\}$ and the straight line with slope -1 joining $\left(e, v^{*}+e\right)$ and $\left(v^{*}+e, e\right)$. (See Fig. 1). The only point on the efficient boundary which is feasible without transfers, in either the one-shot or the repeated game, is $\left(v_{1}^{*}, v_{2}^{*}\right)$, corresponding to the action pair $s^{*}=\left(s_{1}^{*}, s_{2}^{*}\right)$. Our aim is to find efficient SRP equilibria. Clearly, on the equilibrium path of any such equilibrium, $s^{*}$ must be played in every period. If a point such as $B$ in Fig. 1 is to be the equilibrium payoff then one player must also make a transfer to the other player ( 2 must pay 1 in the case of $B$ ). Suppose that player $i$ deviates at some stage. By playing the best response to $s_{j}^{*}$ and declining to pay any transfer she can obtain a single-period payoff of $c_{i}\left(s^{*}\right)+e+\phi_{j}$, where $\phi_{j}$ is the expected transfer from $j$ to $i$. If $s^{*}$ is not a Nash equilibrium of $g$ this is strictly greater (for some $i$ ) than $v_{i}^{*}+e+\phi_{j}$, which implies that the deviation is profitable in the current period. Therefore punishments are required for deviations. Can such punishments be efficient? For example, one might keep playing $s^{*}$ and try to levy larger and larger fines for each transgression. Clearly, this will not work because the player being punished could always do better by not paying the fine: the argument just given shows that in each period player 1 could get at least $c_{1}\left(s^{*}\right)+e$ (if 2 stuck to the equilibrium) and similarly player 2 could get at least $c_{2}\left(s^{*}\right)+e$. Since

$$
c_{1}\left(s^{*}\right)+c_{2}\left(s^{*}\right)+2 e>v^{*}+2 e,
$$

this is impossible. Efficient punishments being impossible, any efficient SRP equilibrium must involve some inefficient continuation payoffs. By the definition of SRP equilibrium, all such continuation payoffs must lie on the Pareto-frontier of $W(\delta)$, the set of WRP payoffs given discount factor $\delta$. As we noted in the Introduction, there are games (such as Bertrand duopoly) where this condition cannot be achieved. The question is: does the additional structure given by side-payments ensure that it can be achieved?

Figures 2 and 3 illustrate two plausible methods of constructing SRP equilibria with payoffs $\left(v_{1}^{*}+e, v_{2}^{*}+e\right)$, and why they will not work in general. In the first 'equilibrium', $\left(s_{1}^{*}, s_{2}^{*}\right)$ is played in the normal phase and there are no transfers. If player 2 deviates, player 1 minmaxes 2 while 2 
Player 2's payoff

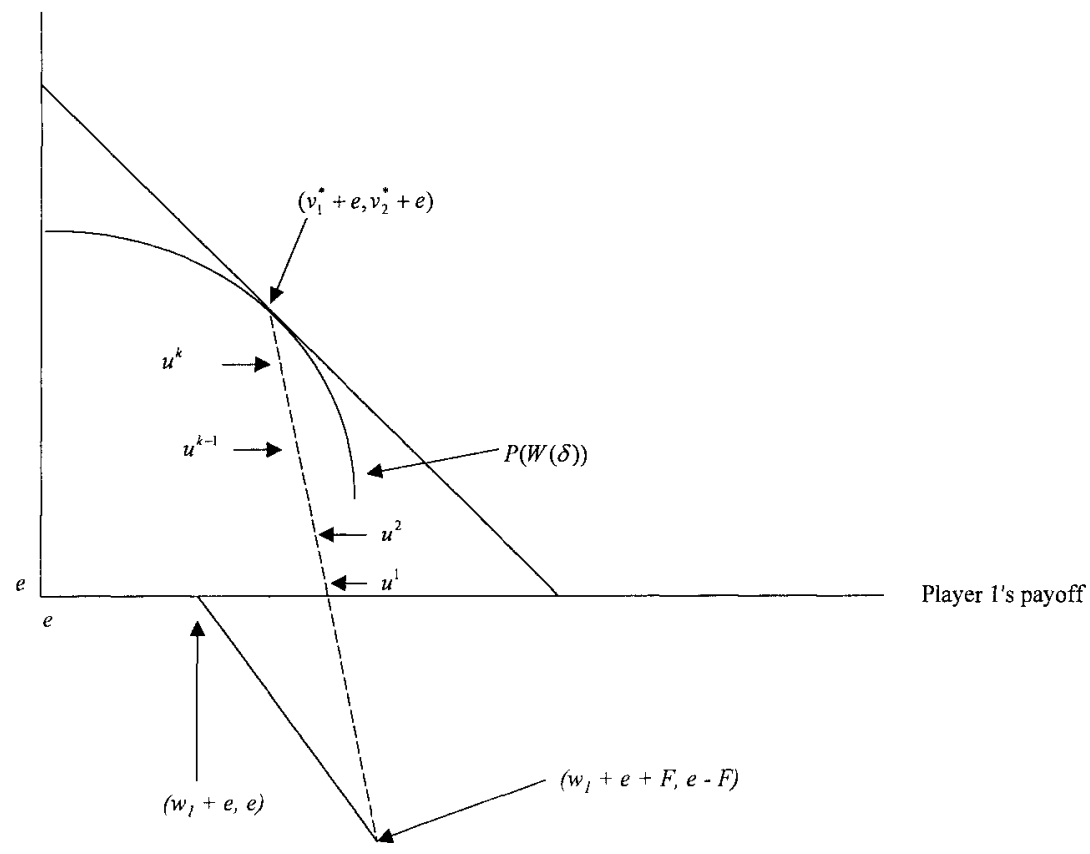

FIGURE 2

plays a best response and pays a fine $F$ to 1 . This punishment phase lasts for $k$ periods and play then reverts to the normal phase. If 2 deviates during the punishment phase then the punishment starts again. A similar punishment sequence is used to deter deviations by player 1 . Figure 2 shows the stage payoff pair during 2's punishment phase excluding the fine $\left(\left(w_{1}+e, e\right)\right)$ and including the fine $\left(\left(w_{1}+e+F, e-F\right)\right)$; it also shows the sequence of continuation payoff pairs, starting from the beginning of 2's punishment phase, $\left(u^{1}, u^{2}, \ldots, u^{k},\left(v_{1}^{*}+e, v_{2}^{*}+e\right)\right)$. The problem with this is that, as shown, the continuation payoffs may not all lie on $P(W(\delta))$. That is, one might be able to construct other WRP equilibria which Pareto-dominate payoffs such as $u^{k}$. In the second "equilibrium," the normal phase action pair is again $\left(s_{1}^{*}, s_{2}^{*}\right)$ with no transfers. In this case, the punishment phase lasts for a single period and the fine, $F$, is large enough to ensure that the continuation payoff during the punishment phase, $u$, is in $P(W(\delta)$ ) (see Fig. 3). This would then be SRP since the only continuation payoffs would be $\left(v_{1}^{*}+e, v_{2}^{*}+e\right), u$ and a point symmetric to $u$ which punishes player 1 . The problem in this case is that, since

$$
u=(1-\delta)\left(w_{1}+e+F, e-F\right)+\delta\left(v_{1}^{*}+e, v_{2}^{*}+e\right),
$$


Player 2's payoff

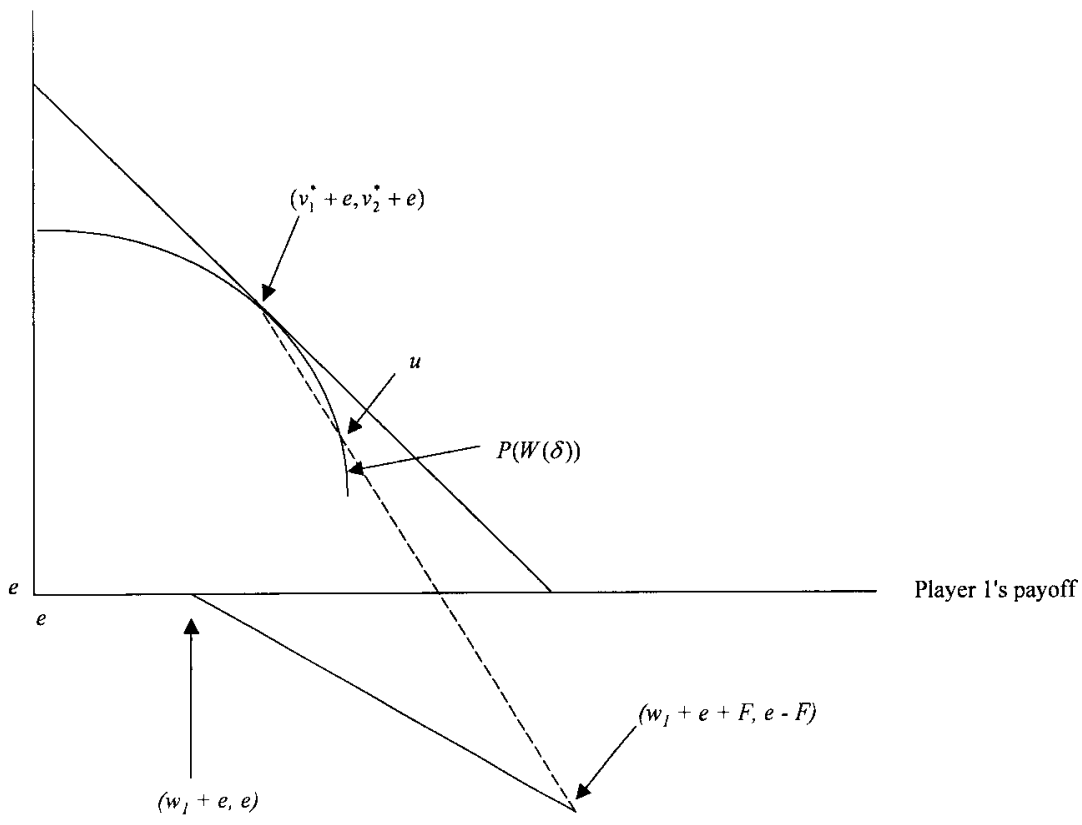

FIGURE 3

the fine needed in the punishment phase may have to become unboundedly large as the discount factor approaches 1 (for given $F, u$ is below the efficient frontier $P\left(V^{*}\right)$ and close to $\left(v_{1}^{*}+e, v_{2}^{*}+e\right)$ if $\delta$ is near 1 , and we show below, in the discussion before Corollary 1 , that any point on $P\left(V^{*}\right)$ is WRP for $\delta$ near 1). Since per-period wealth is bounded this suggests that this approach cannot work for high discount factors.

Before presenting our results, we need to establish some facts about WRP equilibria. Let $\bar{W}(\delta)$ be the closure of $W(\delta)$. Let

$$
\tilde{v}_{1}^{2}(\delta):=\max \left\{v_{1} \mid\left(v_{1}, v_{2}\right) \in \bar{W}(\delta) \text { for some } v_{2}\right\}
$$

and let

$$
\tilde{v}_{2}^{2}(\delta):=\min \left\{v_{2} \mid\left(\tilde{v}_{1}^{2}(\delta), v_{2}\right) \in \bar{W}(\delta)\right\} .
$$

$\left(\tilde{v}_{1}^{2}(\delta), \tilde{v}_{2}^{2}(\delta)\right)$ is the bottom-right point of $P(W(\delta))$ : effectively, player 1's best and player 2's worst WRP payoff, given $\delta$. Let $\left(\tilde{v}_{1}^{1}(\delta), \tilde{v}_{2}^{1}(\delta)\right)$ be, analogously, the top-left point of $P(W(\delta))$. We will sometimes suppress $\delta$ and write $\left(\tilde{v}_{i}^{i}, \tilde{v}_{j}^{i}\right)(i=1,2 ; j \neq i)$ for ease of exposition.

THEorem 1. (Farrell and Maskin, 1989). Let $v=\left(v_{1}, v_{2}\right)$ be in $V^{*}$. If there exist action-pairs $a^{i}=\left(a_{1}^{i}, a_{2}^{i}\right)($ for $i=1,2)$ in $A_{1}^{\prime} \times A_{2}^{\prime}$ such that 
$c_{i}^{\prime}\left(a^{i}\right)<v_{i}$ and $g_{j}^{\prime}\left(a^{i}\right) \geq v_{j}$ for $j \neq i$ then the payoffs $\left(v_{1}, v_{2}\right)$ are WRP for sufficiently large $\delta<1$.

Farrell and Maskin (1989) prove the above result for the case where each player has a finite action set. The generalization to our context, where the set of possible fines is a continuum, is straightforward. The actions $a^{i}$ are used to punish $i$ for deviations from the normal phase actions which generate payoff $v$. The condition $c_{i}^{\prime}\left(a^{i}\right)<v_{i}$ ensures (for high enough $\delta$ ) that the punishment does deter $i$; the condition $g_{j}^{\prime}\left(a^{i}\right) \geq v_{j}$ ensures that the continuations are not Pareto-ranked. Let $\bar{a}^{i}$ be an action pair of $g^{\prime}$ according to which player $j$ min-maxes player $i$ while $i$ plays best response (denote this action pair of $g$ by $\left.a^{i, m}\right)$ and transfers $e$ to $j .\left(\bar{a}^{1}, \bar{a}^{2}\right)$ satisfies the requirements of Theorem 1 for every $v=\left(v_{1}, v_{2}\right) \in V^{*}$ since $c_{i}^{\prime}\left(\bar{a}^{i}\right)=e<v_{i}$ and, by Assumption 3, $e$ is large enough to ensure that $j^{\prime} \mathrm{s}$ payoff $g_{j}^{\prime}\left(\bar{a}^{i}\right)=g_{j}\left(a^{i, m}\right)+2 e>v^{*}+e>v_{j}$. Therefore, for every $v \in V^{*}$ there exists $\delta(v)$ such that $v$ is WRP for all $\delta>\delta(v)$. This establishes the following Corollary, which asserts that the endpoints of $P(W(\delta))$ converge to the endpoints of the efficient frontier $P\left(V^{*}\right)$ as $\delta \rightarrow 1$.

COROLlaRY 1. $\lim _{\delta \rightarrow 1} \tilde{v}_{i}^{i}(\delta)=e$ and $\lim _{\delta \rightarrow 1} \tilde{v}_{i}^{j}(\delta)=v^{*}+e(i=1,2$, $i \neq j)$.

Farrell and Maskin (1989) have a simple sufficient condition for existence of SRP equilibrium (their Theorem 5), but the result above shows that it cannot be satisfied in our side-payments model. Let $\bar{W}$ be the closure of the set of payoff pairs which are WRP for some $\delta<1$ and let $w^{i}$ be the payoff pair which is worst for $i$ in $\bar{W} \cap P\left(V^{*}\right)$. Their sufficient condition is that, for $i=1,2$ and $j \neq i$ there is an action pair $\alpha^{i}$ such that $c_{i}^{\prime}\left(\alpha^{i}\right)<w_{i}^{i}<w_{i}^{j}$ and $g_{j}^{\prime}\left(\alpha^{i}\right) \geq w_{j}^{i}$. The fact that $\lim _{\delta \rightarrow 1} \tilde{v}_{i}^{i}(\delta)=e$ and $\lim _{\delta \rightarrow 1} \tilde{v}_{j}^{i}(\delta)=v^{*}+e$ implies that $\left(e, v^{*}+e\right),\left(v^{*}+e, e\right) \in \bar{W} \cap P\left(V^{*}\right)$ and so $w_{i}^{i}=e$. Therefore, since $i$ 's min-max payoff is $e$, no $\alpha^{i}$ can exist such that $c_{i}^{\prime}\left(\alpha^{i}\right)<w_{i}^{i}$. It follows that we need a different approach.

It can be shown, using a variation of an argument in Farrell and Maskin (1989) (the proof is available on request), that the set of WRP payoffs, given $\delta$, is closed. Therefore, there exists a WRP equilibrium of $g^{\prime}$ with payoffs $\left(\tilde{v}_{1}^{2}(\delta), \tilde{v}_{2}^{2}(\delta)\right)$. Call this equilibrium $\tilde{\sigma}^{2}(\delta)$. Let the mixed strategy pair (of the original game $g$, i.e. not including the transfers, if any) played in period 1 of $\tilde{\sigma}^{2}(\delta)$ be $\left(\tilde{a}_{1}^{2}(\delta), \tilde{a}_{2}^{2}(\delta)\right) \equiv \tilde{a}^{2}(\delta)$. To be more precise, $\tilde{a}_{1}^{2}(\delta)$ is the marginal over $S_{1}$ of the mixed action played by 1 in period 1 of $\tilde{\sigma}^{2}(\delta)$. $\tilde{\sigma}^{1}(\delta)$ and $\tilde{a}^{1}(\delta)$ are defined analogously. The SRP equilibrium which we construct will use the action pairs $\tilde{a}^{1}(\delta)$ and $\tilde{a}^{2}(\delta)$ to punish the players.

Lemma 1. Given any $\delta<1, c_{i}\left(\tilde{a}^{i}(\delta)\right) \leq \tilde{v}_{i}^{i}(\delta)-e(i=1,2)$. 
Proof. We show first that $\tilde{v}_{2}^{2}$ is the worst continuation payoff for player 2 in $\tilde{\sigma}^{2}$. If $v_{2}<\tilde{v}_{2}^{2}$ and $\left(v_{1}, v_{2}\right)$ is a continuation payoff of $\tilde{\sigma}^{2}$, then $v_{1} \geq \tilde{v}_{1}^{2}$, otherwise $\left(\tilde{v}_{1}^{2}, \tilde{v}_{2}^{2}\right)$ would Pareto-dominate $\left(v_{1}, v_{2}\right)$, which would violate the WRP property of $\tilde{\sigma}^{2}$. Therefore, $v_{1}=\tilde{v}_{1}^{2}$ by definition of $\tilde{v}_{1}^{2}$. But then the fact that $v_{2}<\tilde{v}_{2}^{2}$ contradicts the definition of $\tilde{v}_{2}^{2}$.

Now suppose that $c_{2}\left(\tilde{a}^{2}\right)>\tilde{v}_{2}^{2}-e$. Let $\left(\tilde{z}_{1}, \tilde{z}_{2}\right)$ be the continuation payoffs in $\tilde{\sigma}^{2}$ after player 2 , in period 1 , plays the best response to $\tilde{a}_{1}^{2}$ and pays no transfer to player 1 . Then, by the argument above, $\tilde{z}_{2} \geq \tilde{v}_{2}^{2}$. Therefore, by this strategy, player 2 obtains at least

$$
(1-\delta)\left[c_{2}\left(\tilde{a}^{2}\right)+e\right]+\delta \tilde{z}_{2}>\tilde{v}_{2}^{2}
$$

and therefore has a profitable deviation, which is a contradiction. Hence $c_{2}\left(\tilde{a}^{2}\right) \leq \tilde{v}_{2}^{2}-e$ and similarly $c_{1}\left(\tilde{a}^{1}\right) \leq \tilde{v}_{1}^{1}-e$.

Given any four numbers $\left(F_{1}, F_{2}, z_{1}, z_{2}\right)$ with $0 \leq F_{i} \leq e$ and $0 \leq z_{i} \leq v_{i}^{*}$ $(i=1,2)$, define a pair of strategies $\sigma\left(F_{1}, F_{2}, z_{1}, z_{2} ; \delta\right)$ as follows.

Normal phase for player $i(N(i))(i=1,2)$ : "play $\left(s_{1}^{*}, s_{2}^{*}\right)$ and transfer $v_{i}^{*}-z_{i}$ from player $i$ to player $j "$;

Punishment phase for player $i(P(i))(i=1,2)$ : "play $\tilde{a}^{i}(\delta)$ and transfer $F_{i}$ from player $i$ to player $j "$.

Begin in phase $N(1)$. If $N(i)$ or $P(i)$ obtains at stage $t-1$ and either there is no deviation at $t-1$ or both players deviate at $t-1$, then $N(i)$ obtains in period $t$. In any phase, if player $i$ deviates alone at stage $t-1$, then $P(i)$ obtains at $t$.

$\sigma\left(F_{1}, F_{2}, z_{1}, z_{2} ; \delta\right)$ involves efficient normal phases (one for each player) and single-period punishment phases whereby $i$, the player being punished, pays a fine of $F_{i}$ to $j$ and the actions (of $g$ ) are whatever would be played in the first period of $\tilde{\sigma}^{i}(\delta), i$ 's worst equilibrium in $P(W(\delta))$. For example, when 2 is being punished, the payoff pair in period 1 is

$$
\left(g_{1}\left(\tilde{a}^{2}(\delta)\right)+e+F_{2}, g_{2}\left(\tilde{a}^{2}(\delta)\right)+e-F_{2}\right),
$$

the continuation payoff at the start of the next period (entering 2's normal phase $N(2))$ is $\left(v^{*}+e-z_{2}, e+z_{2}\right)$ and the long-run payoff pair starting in period 1 is therefore

$$
(1-\delta)\left(g_{1}\left(\tilde{a}^{2}(\delta)\right)+e+F_{2}, g_{2}\left(\tilde{a}^{2}(\delta)\right)+e-F_{2}\right)+\delta\left(v^{*}+e-z_{2}, e+z_{2}\right) .
$$

Theorem 2 below shows that, given $\delta$ sufficiently high, it is possible to choose $\left(F_{1}(\delta), F_{2}(\delta), z_{1}(\delta), z_{2}(\delta)\right)$ so that $\sigma(\delta) \equiv \sigma\left(F_{1}(\delta), F_{2}(\delta), z_{1}(\delta)\right.$, $\left.z_{2}(\delta) ; \delta\right)$ is SRP. The argument of the proof is that $\sigma(\delta)$ satisfies the following conditions: (i) $\sigma(\delta)$ is subgame-perfect; (ii) no continuation equilibrium of $\sigma(\delta)$ Pareto-dominates any other; and (iii) $i$ 's continuation payoff when $i$ is about to be punished is equal to $\tilde{v}_{i}^{i}(\delta)$, i.e.,

$$
(1-\delta)\left[g_{2}\left(\tilde{a}^{2}(\delta)\right)+e-F_{2}(\delta)\right]+\delta\left[e+z_{2}(\delta)\right]=\tilde{v}_{2}^{2}(\delta),
$$


together with a similar condition for player 1. Conditions (ii) and (iii) are illustrated in Fig. 4. Conditions (i) and (ii) imply that $\sigma(\delta)$ is WRP. The normal-phase continuation payoffs are efficient. Therefore $\sigma(\delta)$ is SRP if neither punishment-phase continuation payoff pair is Pareto-dominated by an element of $W(\delta)$. Now, suppose that $\sigma(\delta)$ is being played and that, at time $t$, play is in phase $P(2)$ (2's punishment phase). By construction, the sum of the payoffs of the two players in period $t$ is equal to the sum of the payoffs in the first period when $\tilde{\sigma}^{2}(\delta)$ is being played. The sum of the continuation payoffs at $t+1$, when $N(2)$ starts, is $v^{*}+2 e$. Since this is the maximum possible joint payoff, it follows that the equilibrium long-run joint payoff of $P(2)$ is at least as high as the equilibrium long-run joint payoff of $\tilde{\sigma}^{2}(\delta)$. The latter is $\tilde{v}_{1}^{2}(\delta)+\tilde{v}_{2}^{2}(\delta)$. Therefore, by (iii) above, player 1 gets a long-run payoff of at least $\tilde{v}_{1}^{2}(\delta)$ in phase $P(2)$ of the strategy profile $\sigma(\delta)$. Since $\tilde{v}_{1}^{2}(\delta)$ is, by definition, the highest payoff which player 1 can get in any WRP equilibrium, we can conclude that, as required, the continuation payoff pair of $P(2)$ is undominated by any element of $W(\delta)$, and the same is true for $P(1)$.

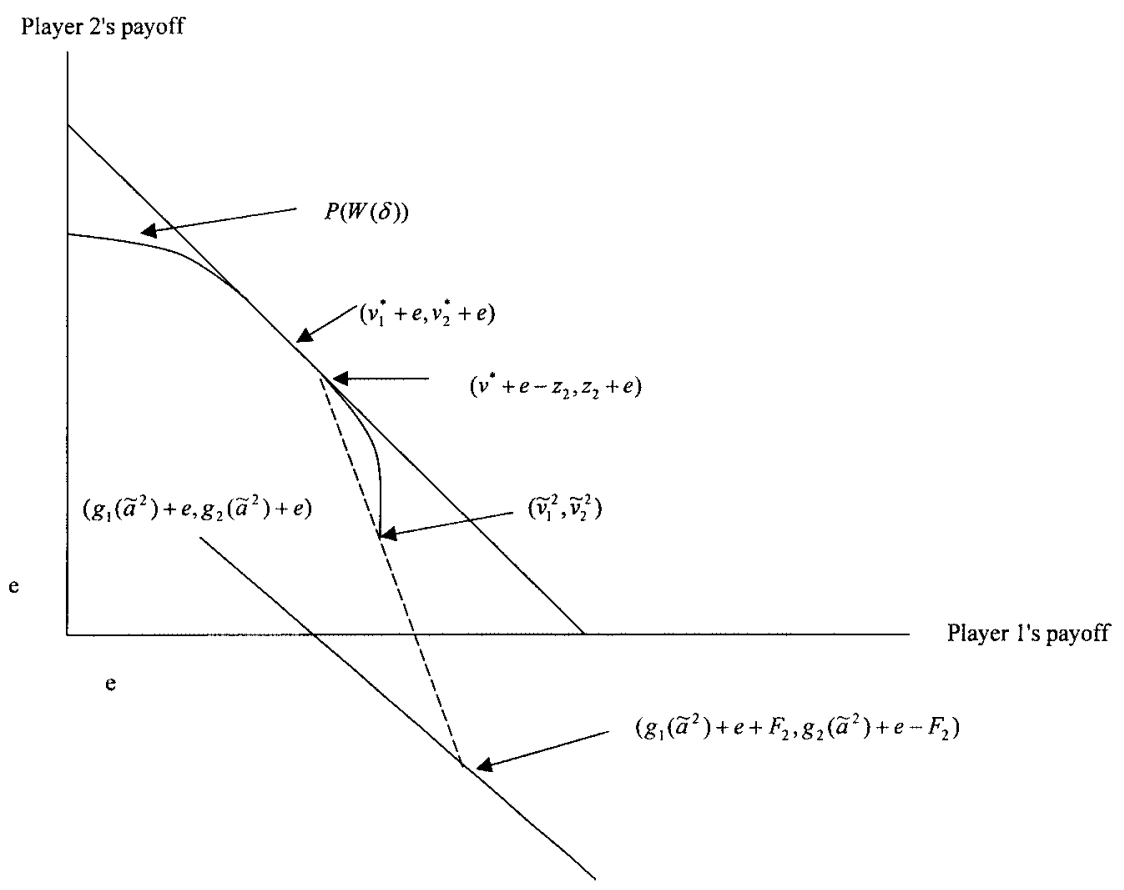

FIGURE 4 
The candidate equilibrium illustrated in Fig. 3 did not work because the fines needed to sustain it become unboundedly large as $\delta \rightarrow 1$. Why does this not happen in the equilibrium $\sigma(\delta)$ ? The reason is that the continuation payoff which player $i$ receives at the end of his punishment period, $z_{i}(\delta)+e$, is very low $\left(z_{i}(\delta) \rightarrow 0\right.$ as $\left.\delta \rightarrow 1\right)$. This means that the continuation payoff at the start of the punishment period can be close to the end of the Pareto boundary $P\left(V^{*}\right)$, despite the bounded fine, and thus undominated by any element of $W(\delta)$.

Let $F_{i}(\delta)$ and $z_{i}(\delta)$, for $i=1,2$, be defined as

$$
\begin{gathered}
F_{i}(\delta):=\max \left[0, v^{*}-g_{j}\left(\tilde{a}^{i}(\delta)\right), c_{i}\left(s^{*}\right)+g_{i}\left(\tilde{a}^{i}(\delta)\right)\right], \\
z_{i}(\delta):=\delta^{-1}\left[\tilde{v}_{i}^{i}(\delta)-e\right]-\delta^{-1}(1-\delta)\left(g_{i}\left(\tilde{a}^{i}(\delta)\right)-F_{i}(\delta)\right) .
\end{gathered}
$$

$z_{i}(\delta)$ is defined in this way so that (1) is satisfied. To motivate the definition of $F_{i}$, note that the condition

$$
F_{i}(\delta) \geq v^{*}-g_{j}\left(\tilde{a}^{i}(\delta)\right)
$$

ensures that player $j$ gets a high payoff when $i$ is being punished (this in turn means that there is no Pareto-ranking among the payoffs). Secondly, the condition

$$
F_{i}(\delta) \geq c_{i}\left(s^{*}\right)+g_{i}\left(\tilde{a}^{i}(\delta)\right)
$$

ensures that $i$ does not find it profitable (assuming $\delta \simeq 1$ ) to deviate during the normal phase when he gets $z_{i}(\delta)+e \simeq e$. The inequality says that the fine outweighs the sum of his incremental payoffs in the current and next periods if he deviates.

Then, for $\sigma(\delta)=\sigma\left(F_{1}(\delta), F_{2}(\delta), z_{1}(\delta), z_{2}(\delta) ; \delta\right)$, we have

THEOREM 2. For all $\delta$ sufficiently high, $\sigma(\delta)$ is a SRP equilibrium.

Proof. We show first that the stipulated transfers are feasible, i.e., that $0 \leq F_{i}(\delta)<e$. Clearly $0 \leq F_{i}(\delta)$. By Assumption 3,

$$
v^{*}-g_{j}\left(\tilde{a}^{i}(\delta)\right)=g_{1}\left(s^{*}\right)+g_{2}\left(s^{*}\right)-g_{j}\left(\tilde{a}^{i}(\delta)\right)<e
$$

and

$$
g_{i}\left(\tilde{a}^{i}(\delta)\right)+c_{i}\left(s^{*}\right)<e .
$$

Next, we show that $z_{i}(\delta) \geq 0$. By definition, $F_{i}(\delta) \geq g_{i}\left(\tilde{a}^{i}(\delta)\right)+c_{i}\left(s^{*}\right)$. Therefore

$$
F_{i}(\delta) \geq g_{i}\left(\tilde{a}^{i}(\delta)\right)-\tilde{v}_{i}^{i}(\delta)+e
$$

(as $\tilde{v}_{i}^{i}(\delta) \geq e$ by individual rationality and as $c_{i}\left(s^{*}\right) \geq v_{i}^{*}>0$ by Assumption 
2), which implies that

$$
\tilde{v}_{i}^{i}(\delta)-e \geq g_{i}\left(\tilde{a}^{i}(\delta)\right)-F_{i}(\delta)
$$

and so $z_{i}(\delta) \geq 0$. By Corollary $1, z_{i}(\delta) \rightarrow 0$ as $\delta \rightarrow 1$. Take $\delta$ sufficiently high that $z_{i}(\delta)<v_{i}^{*}(i=1,2)$.

As noted, $z_{1}(\delta)$ and $z_{2}(\delta)$ are defined so as to satisfy the condition specified in (iii),

$$
(1-\delta)\left[g_{2}\left(\tilde{a}^{2}(\delta)\right)+e-F_{2}(\delta)\right]+\delta\left[z_{2}(\delta)+e\right]=\tilde{v}_{2}^{2}(\delta),
$$

and an equivalent condition for player 1 .

Clearly, $\sigma(\delta)$ has four continuation payoffs. We show next that these are not Pareto-ranked. Player 2's long-run payoffs in the four different subgames are (dropping the $\delta$ arguments for ease of exposition)

$$
\begin{gathered}
P(1):(1-\delta)\left(g_{2}\left(\tilde{a}^{1}\right)+e+F_{1}\right)+\delta\left(v^{*}+e-z_{1}\right), \\
N(1): v^{*}+e-z_{1}, \\
N(2): e+z_{2},
\end{gathered}
$$

and

$$
P(2): \tilde{v}_{2}^{2} \text {. }
$$

By the definition of $c_{2}\left(\tilde{a}^{2}\right)$ and Lemma $1, g_{2}\left(\tilde{a}^{2}\right) \leq c_{2}\left(\tilde{a}^{2}\right) \leq \tilde{v}_{2}^{2}-e$. Therefore, since $F_{2} \geq 0, g_{2}\left(\tilde{a}^{2}\right)+e-F_{2} \leq \tilde{v}_{2}^{2}$. Combining with (2), we have

$$
z_{2}+e \geq \tilde{v}_{2}^{2}
$$

By Corollary $1, \lim _{\delta \rightarrow 1} z_{2}=0=\lim _{\delta \rightarrow 1} z_{1}$ so $v^{*}-z_{1} \geq z_{2}$ if $\delta$ is high enough since $v^{*}>0$. Therefore,

$$
v^{*}+e-z_{1} \geq e+z_{2} .
$$

$g_{2}\left(\tilde{a}^{1}\right)+F_{1} \geq v^{*} \geq v^{*}-z_{1}$ by the definition of $F_{1}$ and the fact that $z_{1} \geq 0$. Thus, $g_{2}\left(\tilde{a}^{1}\right)+e+F_{1} \geq v^{*}+e-z_{1}$. We conclude that

$$
\begin{gathered}
(1-\delta)\left(g_{2}\left(\tilde{a}^{1}\right)+e+F_{1}\right)+\delta\left(v^{*}+e-z_{1}\right) \\
\geq v^{*}+e-z_{1} \geq e+z_{2} \geq \tilde{v}_{2}^{2} .
\end{gathered}
$$

Similar inequalities for player 1 show that player 1 has the opposite ordering over the four continuations and thus that the continuation payoffs are not Pareto-ranked. (See Fig. 4).

We show next that no continuation payoff of $\sigma(\delta)$ is Pareto-dominated by any WRP payoff, given $\delta$ (the following just restates the argument in the text). By (2),

$$
(1-\delta) F_{2}=(1-\delta)\left[g_{2}\left(\tilde{a}^{2}\right)+e\right]+\delta\left[z_{2}+e\right]-\tilde{v}_{2}^{2} .
$$


Therefore, letting $\left(\tilde{y}_{1}, \tilde{y}_{2}\right)$ be the continuation payoffs after the first period of $\tilde{\sigma}^{2}$ (both players having played their equilibrium strategies in the first period),

$$
\begin{aligned}
& (1-\delta)\left(g_{1}\left(\tilde{a}^{2}\right)+e+F_{2}\right)+\delta\left(v^{*}+e-z_{2}\right) \\
= & \left.(1-\delta)\left[g_{1}\left(\tilde{a}^{2}\right)+g_{2}\left(\tilde{a}^{2}\right)+2 e\right]\right)+\delta\left[v^{*}+2 e\right]-\tilde{v}_{2}^{2} \\
\geq & (1-\delta)\left[g_{1}\left(\tilde{a}^{2}\right)+g_{2}\left(\tilde{a}^{2}\right)+2 e\right]+\delta\left(\tilde{y}_{1}+\tilde{y}_{2}\right)-\tilde{v}_{2}^{2} \\
= & \left(\tilde{v}_{1}^{2}+\tilde{v}_{2}^{2}\right)-\tilde{v}_{2}^{2} \\
= & \tilde{v}_{1}^{2}
\end{aligned}
$$

since the sum of the payoffs in the first period of $\tilde{\sigma}^{2}$ is $g_{1}\left(\tilde{a}^{2}\right)+g_{2}\left(\tilde{a}^{2}\right)+2 e$ and the sum of the long-run payoffs from $\tilde{\sigma}^{2}$ is $\tilde{v}_{1}^{2}+\tilde{v}_{2}^{2}$. Therefore, by definition of $\tilde{v}_{1}^{2}$, player 1's payoff in phase $P(2)$ is at least as great as that from any WRP equilibrium. Hence, long run payoffs from $P(2)$ are not Pareto-dominated by those from any WRP equilibrium. A similar argument for phase $P(1)$ and the fact that normal phase payoffs are on the efficient frontier establish that $\sigma(\delta)$ is a SRP equilibrium if it is a subgame perfect equilibrium.

To prove the latter true, for high $\delta$, it suffices, by symmetry, to consider $N(2)$ and $P(2)$. Consider $N(2)$ first and suppose that player 2 deviates. Player 2's maximum deviation payoff is $(1-\delta)\left[c_{2}\left(s^{*}\right)+e\right]+\delta \tilde{v}_{2}^{2}$ since the best that 2 can do if he deviates is play best response to $s^{*}$ and not pay the transfer $v_{2}^{*}-z_{2}$. The payoff from conforming is $z_{2}+e$. The definition of $z_{2}$ implies that $z_{2} \geq \tilde{v}_{2}^{2}-e-(1-\delta)\left[g_{2}\left(\tilde{a}^{2}\right)-F_{2}\right]$. This, together with the fact (from its definition) that $F_{2} \geq g_{2}\left(\tilde{a}^{2}\right)+c_{2}\left(s^{*}\right)$, gives

$$
\begin{aligned}
z_{2}+e & \geq \tilde{v}_{2}^{2}-(1-\delta)\left[g_{2}\left(\tilde{a}^{2}\right)-F_{2}\right] \\
& \geq \tilde{v}_{2}^{2}+(1-\delta) c_{2}\left(s^{*}\right) \\
& =(1-\delta)\left[c_{2}\left(s^{*}\right)+\tilde{v}_{2}^{2}\right]+\delta \tilde{v}_{2}^{2} \\
& \geq(1-\delta)\left[c_{2}\left(s^{*}\right)+e\right]+\delta \tilde{v}_{2}^{2}
\end{aligned}
$$

as $\tilde{v}_{2}^{2} \geq e$ by individual rationality. Therefore, there is no incentive for player 2 to deviate in $N(2)$. Player 1's maximum deviation payoff in $N(2)$ is

$$
(1-\delta)\left(c_{1}\left(s^{*}\right)+e+v_{2}^{*}-z_{2}\right)+\delta \tilde{v}_{1}^{1},
$$

which tends to $e$ as $\delta \rightarrow 1$ by Corollary 1 . The payoff from conforming is $v^{*}+e-z_{2}$ which converges to $v^{*}+e>e$ as $\delta \rightarrow 1$. Hence, for high $\delta$, deviation is not profitable. Now consider phase $P(2)$. Player 2's maximum deviation payoff is

$$
(1-\delta)\left[c_{2}\left(\tilde{a}^{2}\right)+e\right]+\delta \tilde{v}_{2}^{2} \leq \tilde{v}_{2}^{2}
$$


by Lemma 1 . The payoff from conforming is $\tilde{v}_{2}^{2}$ so there is no incentive for player 2 to deviate. Player 1's maximum deviation payoff in $P(2)$ is

$$
(1-\delta)\left(c_{1}\left(\tilde{a}^{2}\right)+e+F_{2}\right)+\delta \tilde{v}_{1}^{1},
$$

which tends to $e$ as $\delta \rightarrow 1$. The payoff from conforming is

$$
(1-\delta)\left(g_{1}\left(\tilde{a}^{2}\right)+e+F_{2}\right)+\delta\left(v^{*}+e-z_{2}\right),
$$

which is greater than $v^{*}+e-z_{2}$ by (3). Since this tends to $v^{*}+e>e$ we conclude, again, that, for high enough $\delta$, no deviation is profitable.

Theorem 2 shows that, for any high discount factor, there exists an SRP equilibrium (which depends on the discount factor). It is easy to see that any point on the line segment between $\left(z_{1}+e, v^{*}+e-z_{1}\right)$ and $\left(v^{*}+e-\right.$ $\left.z_{2}, z_{2}+e\right)$ can also be achieved in SRP equilibrium. Simply play $s^{*}$, with the appropriate transfer; punish a deviation by $i(i=1,2)$ by switching to $P(i)$ as defined in $\sigma(\delta)$ and continue as in $\sigma(\delta)$. Since $\lim _{\delta \rightarrow 1} z_{i}=0(i=1,2)$ this leads to the following result.

COROLLARY 2. The set of SRP payoffs converges to the individually rational, efficient frontier as $\delta \rightarrow 1$.

Therefore we conclude that the efficiency principle holds in the sense that, for high $\delta$, every SRP equilibrium is approximately efficient. Moreover, for every efficient, strictly individually rational payoff $v$ there is a critical value $\delta(v)$ such that $v$ is an SRP payoff if $\delta>\delta(v)$. Notice that, as long as the efficient action pair $\left(s_{1}^{*}, s_{2}^{*}\right)$ is not a Nash equilibrium of $g$, there must exist inefficient SRP equilibria (or, to put it another way, every SRP equilibrium must have inefficient continuation equilibria); furthermore, each player's most preferred equilibrium is inefficient, so that if, ex ante, all the bargaining power is held by one player, the Coase theorem will not apply. Nevertheless, a weaker version of the Theorem does apply because, if the discount factor is high, $\left(s_{1}^{*}, s_{2}^{*}\right)$ will be played with high probability in a high proportion of periods in any SRP equilibrium. This follows from Corollary 2 and the fact that $g$ is finite.

\section{REFERENCES}

Abreu, D., Pearce D., and Stacchetti, E. (1993). "Renegotiation and Symmetry in Repeated Games," J. Econ. Theory 60, 217-240.

Asheim, G. B. (1991). "Extending Renegotiation-Proofness to Infinite Horizon Games," Games Econom. Behavior 3, 278-294.

Bernheim, B. D., and Ray, D. (1989). "Collective Dynamic Consistency in Repeated Games," Games Econom. Behavior 1, 295-326. 
Farrell, J. and Maskin, E. (1989). "Renegotiation in Repeated Games," Games Econom. Behavior 1, 327-360.

Greif, A. (1989). "Reputation and Coalitions in Medieval Trade," J. Econ. History 49, 857-882. Hart, O. (1995). Firms, Contracts and Financial Structure. Oxford: Oxford University Press.

Milgrom, P., and Roberts. J. (1992). Economics, Organization and Management. London: Prentice Hall.

Milgrom, P., North, D, and Weingast, B. (1990). "The Role of Institutions in the Revival of Trade: The Medieval Law Merchant," Econ. and Politics 2, 1-23.

Pearce, D. (1987). "Renegotiation-Proof Equilibria: Rationality and Intertemporal Cooperation," mimeo, Yale University.

Rotemberg, J., and Saloner, G. (1986). "A Supergame-Theoretic Model of Price Wars During Booms," Amer. Econ. Rev. 76, 390-407. 